\title{
Self-consistent analysis of a quantum capacitor
}

\author{
Pawel Pomorski, Hong Guo, and R. Harris \\ Center for the Physics of Materials and Department of Physics, McGill University, Montreal, PQ, Canada H3A 2T8 \\ Jian Wang \\ Department of Physics, The University of Hong Kong, Pokfulam Road, Hong Kong, China
}

(Received 6 May 1998)

\begin{abstract}
We analyze the behavior of the magnetocapacitance for a three-probe capacitor. The self-consistent evaluation of the internal potential is found to play a large role in determining quantitative values of the capacitance. For capacitor plates of mesoscopic size, this potential reduces the charge accumulation by more than an order of magnitude compared to that obtained with noninteracting models. However, the qualitative behavior of the magnetocapacitance is not substantially altered by the self-consistency. A simple but physically motivated model gives an analytical formula which compares well with the numerical data. [S0163-1829(98)05347-8]
\end{abstract}

It has been known for many years that quantum effects can influence the behavior of a capacitor. ${ }^{1}$ Classically, capacitance is obtained by solving an electrostatic problem, determining the small bias voltage difference, $\Delta V$, which is needed to transfer a charge $\Delta Q$ between two conductors. The electrostatic capacitance $C_{e}=\Delta Q / \Delta V$ is geometrical: it only depends on the geometric properties of the two conductors. However, early studies of quantum corrections to $C_{e}$ focused on the fact that the Fermi energy $E_{F}$ of the conductors changes with the bias voltage, so that an experimentally measured capacitance should reflect this quantum effect. It was found ${ }^{1}$ that this density of states (DOS) correction is in "series connection" to $C_{e}$, so that the total capacitance is given by $1 / C=1 / C_{e}+\sum_{i} 1 /\left(e^{2} D_{i}\right)$, where $i=1,2$ denotes the two conductors, and $D_{i}=d N_{i} / d E$ is the DOS of conductor $i$ at its Fermi energy.

More recent studies of quantum effects to capacitance have focused on wave phenomena: ${ }^{2}$ for mesoscopic or microscopic conductors, quantum coherence of the entire capacitor including the leads can be maintained. Hence the experimentally measured capacitance becomes an electrochemical one, ${ }^{2} C=e \Delta Q / \Delta \mu$, where $\Delta \mu$ is the electrochemical potential variation between the two electron reservoirs which are connected to the two capacitor plates. This naturally leads to a dynamic perspective on capacitance: ${ }^{3} C$ measures the dynamic charge response when the chemical potential of a reservoir is changed by a small amount. The consequences of wave phenomena for capacitance are various, including, for example, the role played by the leads, which limits the transport modes, ${ }^{3,4}$ the symmetries of the capacitance matrix, ${ }^{5}$ and the relationship to quantum chaotic scattering in mesoscopic cavities. ${ }^{6}$

An important physical ingredient in the case of very small capacitor plates is the electron-electron interaction. ${ }^{2}$ For macroscopic metal plates this interaction is largely screened, but for tiny plates where the DOS is low, the screening length can be long enough to play an important role. From the point of view of ac quantum transport, the interaction is also needed to maintain charge and current conservation. ${ }^{2}$ The simplest way to introduce the interaction is through an RPA-type treatment which is adequate for the capacitor problems $^{2}$ studied so far. Nevertheless, such an analysis involves the self-consistent solution of quantum scattering together with the Poisson equation for the potential distribution in the capacitor, and thus can be complicated for practical device geometries.

It is easy to see why it is necessary to solve a selfconsistent problem. ${ }^{2}$ When the chemical potential of a reservoir is suddenly changed by $\Delta \mu$ from equilibrium, charge with density $\Delta \rho_{\text {inj }}$ is injected into the capacitor. The value of the injected charge can be determined by solving a quantum scattering problem. However, because of the electronelectron interaction, an induced charge density $\Delta \rho_{\text {ind }}(\mathbf{r})$ is established inside the capacitor to oppose the injection. The total charge density of the system is thus given by $\Delta \rho(\mathbf{r})$ $=\Delta \rho_{\text {inj }}+\Delta \rho_{\text {ind }}$. It is this induced charge density which is self-consistently determined by iterating the Poisson equation for the potential distribution $U(\mathbf{r})$ inside the system. The total charge on a plate is then obtained as $\Delta Q=\int \Delta \rho(\mathbf{r}) d \mathbf{r}$, where the integral is carried out over the entire volume of the plate, and the capacitance is defined as $C=e \Delta Q / \Delta \mu$.

This Brief Report examines the quantitative effect of the self-consistent interaction on the behavior of magnetocapacitance for a three-probe mesoscopic capacitor. The same system was used previously to examine the symmetry properties of the capacitance ${ }^{7}$ when the applied external uniform magnetic field $\mathbf{B}$ is reversed. Because the qualitative magneticfield symmetry properties do not depend on the electronelectron interaction in an essential way, Ref. 7 resolved the technical difficulties of the complete self-consistent problem by using classical image charges and then correcting the injectivity. While this procedure is acceptable for qualitative discussion of the problem, the image charges are not adequate to account quantitatively for the induced charge. Therefore, this paper focuses on the quantitative contribution of the full self-consistent cycle to the values of $C=C(\mathbf{B})$ : to the best of our knowledge, this important information has never previously been documented for practical calculations of finite-sized mesoscopic conductors.

To be specific, the three-probe device represented by the white lines in Fig. 1 consists of a two-probe semiconductor plate of size $3300 \AA \times 3300 \AA$, connected to reservoirs 1 and 


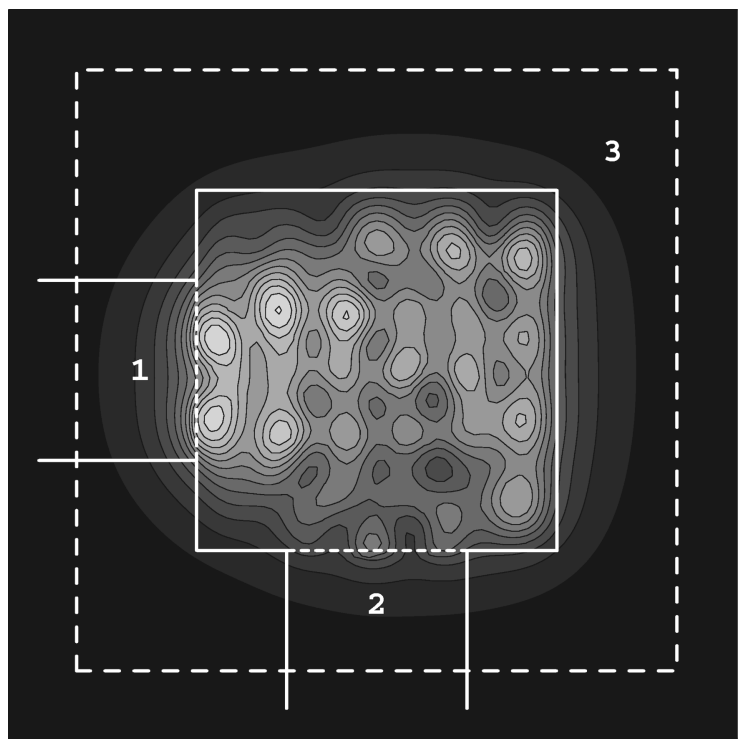

FIG. 1. Contour plot of the characteristic potential $u_{1}(\mathbf{r})$ for a plane parallel to the plate and inside it, and for a magnetic field $\mathbf{B}$ $=\mathbf{0}$. The contour lines are in steps of 0.05 , up to a maximum of 0.65 . The solid white square indicates the position of the plate and its leads, and the dashed square indicates the position of the gate.

2 through quantum wires both of width $1650 \AA$. The scattering potential of the system is specified as $V(\mathbf{r})=0$ everywhere except at the boundaries, where it is assumed to be infinite.

In the vertical direction the plate is $260 \AA$ thick-a reasonable value for confined electrons in heterostructures, and rather smaller than the screening length. The second capacitor plate is a metal gate on top of and parallel to the semiconductor plate: it is attached to a reservoir by the third probe, as shown. The gate has a cross section of $5500 \AA$ $\times 5500 \AA$, similar in size to the plate, but in the vertical direction it is much thicker-many times the screening length, so that the interaction is completely screened deep inside the gate along this direction. Between the two conductors there is an insulating layer with thickness $d=360 \AA$. All space surrounding the plates has a uniform dielectric constant $\epsilon=13.1$ typical of heterostructures.

The calculation proceeds using the theoretical development of Ref. 2. The injected density from the $i$ th probe is calculated $^{8,9}$ from the injectivity $d n(\mathbf{r}, i) / d E$, which is defined in terms of the scattering wave functions $\Psi_{m}$ of the free electrons incoming from probe $i: \quad d n(\mathbf{r}, i) / d E$ $=\Sigma_{m}\left|\Psi_{m}(\mathbf{r})\right|^{2} / h v_{m}$. Here $v_{m}$ is the speed of the incoming wave with mode index $m$, and $h$ is the Planck constant. Thus the injected charge is $\Delta \rho_{\text {inj }}(\mathbf{r})=\Sigma_{i}[d n(\mathbf{r}, i) / d E] \Delta \mu_{i}$. The induced charge due to injection through a probe $i$ is given by $\Delta \rho_{\text {ind }}(\mathbf{r})=-\int \Pi\left(\mathbf{r}, \mathbf{r}^{\prime}\right) e U\left(\mathbf{r}^{\prime}\right) d \mathbf{r}^{\prime}$, where the Lindhard function $\Pi\left(\mathbf{r}, \mathbf{r}^{\prime}\right)$, in the Thomas-Fermi linear screening model, is the sum of emissivities, ${ }^{2} \Pi\left(\mathbf{r}-\mathbf{r}^{\prime}\right)=\delta$ $\left(\mathbf{r}-\mathbf{r}^{\prime}\right) \sum_{i} d n(i, \mathbf{r}) / d E$. For small $\Delta \mu_{i}$, the internal potential $U(\mathbf{r})$ can be written as $e U(\mathbf{r})=u_{i}(\mathbf{r}) \Delta \mu_{i}$, in terms of a characteristic potential $u_{i}(\mathbf{r})$ satisfying a Poisson equation, ${ }^{2}$

$$
-\epsilon \nabla^{2} u_{i}(\mathbf{r})+4 \pi e^{2}\left[\sum_{j} \frac{d n(j, \mathbf{r})}{d E}\right] u_{i}(\mathbf{r})=4 \pi e^{2} \frac{d n(\mathbf{r}, i)}{d E} .
$$

When the magnetic field $\mathbf{B}$ is not zero, the injectivity $d n(\mathbf{r}, j) / d E$ does not equal the emmisivity $d n(j, \mathbf{r}) / d E$ for a conductor with more than one probe, but the sums over the indices $j$ are equal. ${ }^{9}$ Thus the emissivities in Eq. (1) can be replaced with injectivities evaluated in terms of the scattering wave function discussed above. Once $u_{i}(\mathbf{r})$ is obtained from Eq. (1), the electrochemical capacitance ${ }^{2}$ becomes $C_{k l}$ $=-e^{2} \int\left[u_{l}(\mathbf{r}) d n(\mathbf{r}, k) / d E\right] d \mathbf{r}$ for $k \neq l$.

To carry out the calculation described in the preceding paragraph, some technical difficulties must be solved and certain approximations made. The quantum scattering problem is solved using a finite element numerical method documented in Ref. 10 with the Fermi energy chosen to include two modes. It involves no particular complications. However, the numerical solution of Eq. (1) is more involved. The difficulties that arise are the same as those for the computation of capacitance for a system of conductors which is enclosed in a large 3D volume $\Omega$. In this situation, even if $\Omega$ is so large that its surface is far away from all interior charges, the solution of the strictly classical Poisson problem shows that there are induced charges on its surface, so that the definition of capacitance requires considerable care. ${ }^{12}$ Roughly speaking, the relative magnitude of the charge on the surface of $\Omega$ is a measure of the corrections to the conventional classical definition. We will assume that similar arguments apply in the quantum case.

Fortunately, however, the capacitance-at least classically-depends only upon the interaction between specific portions of the system and not upon the details of the boundary conditions at infinity. Thus, in our situation, it is convenient to replace the real configuration by one where the plate and gate are enclosed by grounded metal shields. The reservoirs are outside these shields, and the leads enter them through small holes. In the solution of Eq. (1), the potentials $u_{i}(\mathbf{r})$ can then be locally determined by neglecting the reservoirs and leads, and retaining only the plate and gate densities.

The grounded shields define a "solution box" whose size is chosen to be large enough that the potential on its boundary can be safely set to zero (mimicking $\mathbf{r} \rightarrow \infty$ ). The box was chosen as $8800 \AA \times 8800 \AA \times 5120 \AA$. For such a large box, a conventional relaxation method to solve Eq. (1) is impractical, because it requires a prohibitively large amount of computer time, and a multigrid method ${ }^{11}$ was therefore developed.

After the $u_{i}(\mathbf{r})$ have been obtained, a crucial numerical check is to verify the overall charge distribution of the system. As discussed above, it is not necessary that the charges on the gate and on the plate be equal in value and opposite in sign, but it is important to know the relative magnitude of their difference in charge. For the chosen box size, the charges on the gate and the plate balanced to within $20 \%$, so that we estimate our capacitance calculations to be reliable also to within $20 \%$.

A typical profile of the characteristic potential $u_{1}(\mathbf{r})$ for chemical potentials $\Delta \mu_{1} \neq 0$ and $\Delta \mu_{i}=0$ for $i=2,3$ is shown in Figs. 1 and 2. The presence of two densities of states from the two contacts means that the electrostatic potential has to be highly nonuniform in order for the injected charge to be uniformly screened. It is also the source of the valleys in the characteristic potential. Thus the self-consistent first-order 


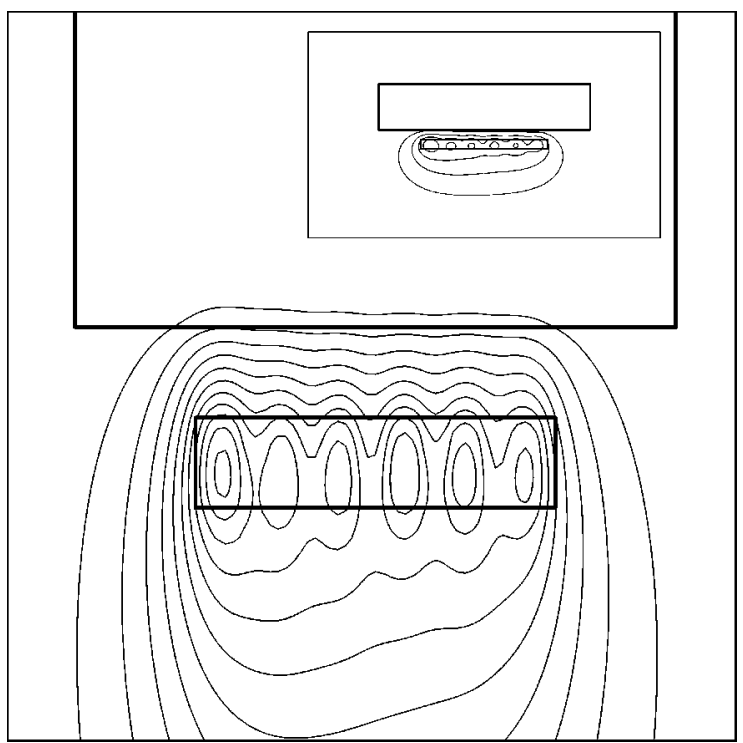

FIG. 2. Contour plot of the characteristic potential $u_{1}$ (r) (not to scale) for a plane perpendicular to the plate at magnetic field $\mathbf{B}$ $=\mathbf{0}$. The contour lines are in steps of 0.05 , up to a maximum of 0.55 . The solid heavy lines correspond to the boundaries of the plate and the gate. Inset: the same plot, to scale, showing the entire computational cell and the positions of the conductors inside it.

potential response introduces a complicated, highly nonuniform modification to the scattering potential, even for an open ballistic system. The plot in Fig. 2 also illustrates the limitations of leaving out of consideration the charge in the leads. The drops in the potential at the junctions where the leads are attached are unphysical. However, a cut has to be imposed somewhere because it is difficult explicitly to model the reservoir and its connection to the lead.

The numerical results for the capacitance $C_{31}$ as a function of an external uniform magnetic field $\pm \mathbf{B}$, applied perpendicular to the plane of Fig. 1, are shown as solid lines in Figs. 3 and 4. The two figures differ only in direction of the field $\mathbf{B}$. Several observations are in order. First, it is evident that $C_{31}(\mathbf{B}) \neq C_{31}(-\mathbf{B})$ : this is the asymmetry anticipated from a general argument based on ac transport theory. ${ }^{2}$ It is experimentally and theoretically confirmed in Ref. 5, and numerically verified in Ref. 7. Second, the near regularly spaced peaks when $|\mathbf{B}|>5000 \mathrm{G}$ are due to Aharonov-Bohm effects as discussed in Ref. 7. These qualitative results are not affected by the self-consistent calculation of the induced charge density. Third, and most important, is that the inclusion of self-consistency drastically reduces the values of $C_{31}$ by about a factor of ten. The reason is related to the reduction of the accumulated charge on the plate (and the gate): $\Delta \rho(\mathbf{r})$ is much smaller than the injected $\Delta \rho_{\text {inj }}(\mathbf{r})$ because the induced charge $\Delta \rho_{\text {ind }}(\mathbf{r})$ cancels a large part of the injected charge at each local point. For our particular system this reduces the total charge to about $10 \%$ of the injected value.

Using the numerically determined local DOS (injectivity and emissivity), it is also possible to compute the entire $C_{31}$ (B) curves analytically after making some approximations. For this purpose, Eq. (1) is reduced to 1D by averaging the local DOS in the $x-y$ plane. Furthermore, since the plate is thin, it may be treated as an infinitesimally thin sheet of charge, just as the metal gate becomes an infinitely long rod

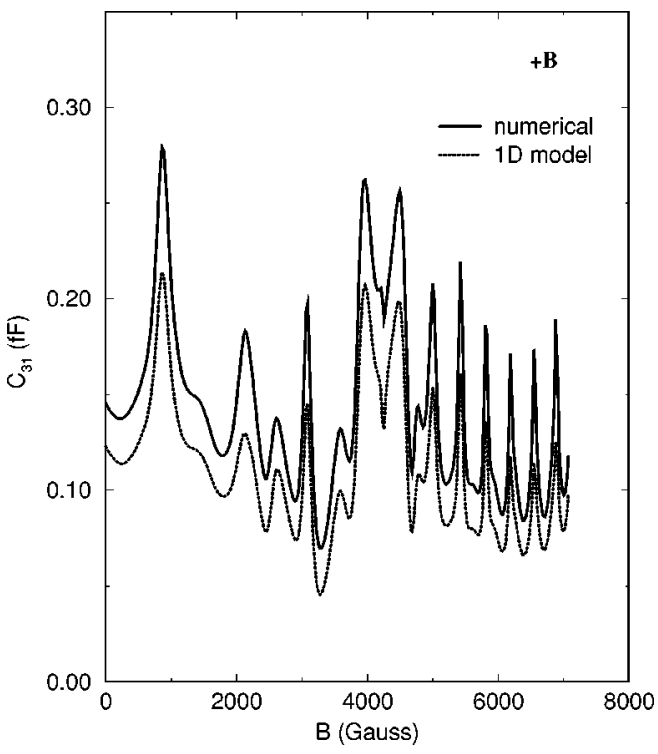

FIG. 3. Capacitance $C_{31}$ as a function of the applied uniform magnetic field B. Solid line: from full self-consistent numerical analysis; dotted line: from the one-dimensional model calculation with the numerical density of states as input parameters. The reasonable agreement of the two curves is clear.

with cross-sectional area $A$. Since the distance $d$ between the two conductors is small compared with the linear size of the plate, fringing fields can be neglected. The probes extend to $\mathbf{r}=\infty$, and thus the boundary conditions are $u_{i}(\mathbf{r})=1$ when $\mathbf{r}$ is inside a lead $i$, and zero otherwise. The 1D Poisson equation is then solved in the same spirit as that of Ref. 2 by matching the solutions of $u_{1}(z)$ across the thin sheet of charge. It is not difficult to obtain the formula

$$
C_{31}=\frac{\epsilon A}{4 \pi} \frac{\lambda_{12}}{\lambda_{1}} \frac{1}{d+\epsilon\left(\lambda_{3}+\lambda_{12}\right)},
$$

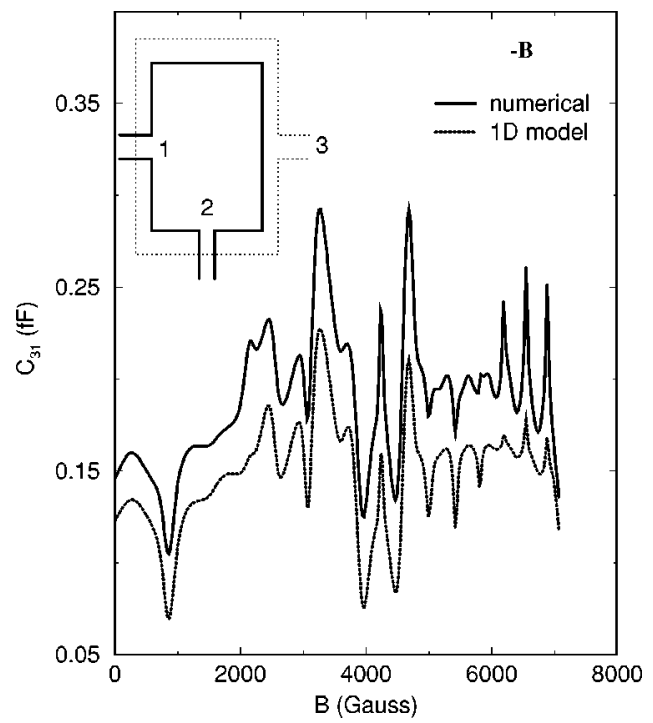

FIG. 4. Capacitance $C_{31}$ when the direction of applied uniform magnetic field is reversed to $-\mathbf{B}$. Solid line: from full selfconsistent numerical analysis; dotted line: from the onedimensional model calculation with the numerical density of states as input parameters. 
where $A=(3300 \AA)^{2}$ is the plate area. The lengths $\lambda$ are defined as $\lambda_{i}=\left[4 \pi e^{2} d \sigma_{i} / d E\right]^{-1}$ for $i=1,2$, $\lambda_{12}=\left[4 \pi e^{2} \sum_{i=1}^{2} d \sigma_{i} / d E\right]^{-1}$, and $\lambda_{3}=\left[4 \pi e^{2} d n_{3} / d E\right]^{-1 / 2}$. The quantities $d \sigma_{i} / d E=\int[d n(\mathbf{r}, i) / d E] d \mathbf{r}$ for $i=1,2$ are the average DOS per unit area for the sheet of charge (the integration is over a unit area), and $d n_{3} / d E$ is the average DOS per unit volume for the gate. These quantities can be obtained from the numerical solutions of the self-consistent problem. The approximate results from Eq. (2) are plotted in Figs. 3 and 4 as the dotted lines, and are seen to reproduce the numerical results remarkably well. The simple analytical formula (2) allows us to observe that the peaks in the capacitance curves are due to the peaks in the injectivity from lead 1 , while the valleys correspond to values of the field at which there are peaks in the injectivity from reservoir 2. The approximate results (2) consistently underestimate the values of $C_{31}(\mathbf{B})$, due, it seems, to the neglect of the fringing fields and to the assumption of a constant local DOS throughout the metal gate.

Finally, it is interesting to discuss the classical limit of the capacitor. If the potential of probe 1 on the plate is raised to $V$ while keeping the other probes grounded, then a current will flow from probe 1 to 2 . Along the way there is no resistance anywhere except at the junction between the probes and the plate. Assuming these probes are identical, there are thus two equivalent resistors, with a voltage drop of $V / 2$ on each. Hence, the classical plate, with an infinite DOS and zero screening length (the $\lambda$ 's), must become an equipotential at $V / 2$. The classical "electrochemical" capacitance of the system is thus $C_{e} / 2$, where $C_{e} \equiv \epsilon A /(4 \pi d)$ is the classical electrostatic capacitance of a parallel plate capacitor. Here we put the word electrochemical in quotes because such a concept makes no sense in classical physics: it merely means that we measure the potential drop from the reservoirs while the drop across the two conductors can be quite different. The quantum formula of Eq. (2) reproduces this conclusion: letting the DOS become infinite yields $d \gg \epsilon\left(\lambda_{3}+\lambda_{12}\right)$ and $\lambda_{12}=\lambda_{1} / 2$, which gives $C_{31}=C_{e} / 2$.

To summarize, the self-consistent solution of the internal potential distribution plays an important role in determining the capacitance of a mesoscopic capacitor. For the small system used as an example, the induced charge reduces the total accumulated charge to about one-tenth of the injected value, thus affecting the value of $C$ in a drastic way. Other symmetry properties of the capacitance matrix do not change qualitatively as a result of the self-consistency. A surprising result is that formula (2), obtained from a crude analysis, when evaluated with the computed density of states, reproduces the full numerical solutions quite well, in spite of the high degree of nonuniformity of the potential distribution.

We gratefully acknowledge support by the NSERC of Canada, the FCAR of Québec, and the RGC of the SAR Government of Hong Kong under Grant No. HKU 7112/97P.
${ }^{1}$ T. P. Smith III, B. B. Goldberg, P. J. Stiles, and M. Heiblum, Phys. Rev. B 32, 2696 (1985); T. P. Smith III, W. J. Wang, and P. J. Stiles, ibid. 34, 2995 (1986).

${ }^{2}$ M. Büttiker, H. Thomas, and A. Prêtre, Phys. Lett. A 100, 364 (1993); M. Büttiker, J. Phys.: Condens. Matter 5, 9361 (1993).

${ }^{3}$ T. Christen and M. Büttiker, Phys. Rev. Lett. 77, 143 (1996).

${ }^{4}$ Jian Wang et al., Phys. Rev. Lett. 80, 4277 (1998).

${ }^{5}$ W. Chen, T. P. Smith III, M. Büttiker, and M. Shayegan, Phys. Rev. Lett. 73, 146 (1994); T. Christen and M. Büttiker, Phys. Rev. B 55, R1946 (1997).

${ }^{6}$ V. A. Gopar, P. A. Mello, and M. Büttiker, Phys. Rev. Lett. 77, 3005 (1996).
${ }^{7}$ H. Wei, N. Zhu, J. Wang, and H. Guo, Phys. Rev. B 56, 9657 (1997).

${ }^{8}$ M. Büttiker and T. Christen, in Quantum Transport in Semiconductor Submicron Structures, edited by B. Kramer (Kluwer Academic Publishers, Dordrecht, 1996).

${ }^{9}$ V. Gasparian, T. Christen, and M. Büttiker, Phys. Rev. A 54, 4022 (1996).

${ }^{10}$ Yongjiang Wang, Jian Wang, and Hong Guo, Phys. Rev. B 49, 1928 (1994).

${ }^{11}$ A. Brandt, Math. Comput. 31, 333 (1977).

${ }^{12}$ Eugene W. Cowan, Basic Electromagnetism (Academic Press, New York, 1968), pp. 419. 